\title{
Zusammenfassung der Überarbeitungen 2019
}

\author{
Yvonne Winhofer-Stöckl · Martin Clodi · Alexandra Kautzky-Willer
}

Die Diabetestherapie unterliegt einem raschen Wandel, da laufend neue wissenschaftliche Erkenntnisse und technologische Innovationen veröffentlicht werden, die Eingang in die therapeutischen Entscheidungen finden. Deshalb müssen auch Empfehlungen für die klinische Praxis regelmäßig nicht nur in ihrem Inhalt, sondern auch Aufbau und Schwerpunkten adaptiert werden. Diese „Zusammenfassung der Überarbeitungen 2019“ soll Ihnen einen ersten Überblick über die Revisionen der vorherigen Leitlinien aus dem Jahr 2016 geben.

\section{Diabetes mellitus - Definition, Klassifikation, Dia- gnose, Screening und Prävention}

Die vormals geteilten Leitlinien wurden zu einer Leitlinie zusammengefasst. Empfehlungen zum Screening auf Typ-2-Diabetes, aber auch auf seltene Formen

\footnotetext{
Y. Winhofer-Stöckl (凶)

Klinische Abteilung für Endokrinologie und Stoffwechsel, Universitätsklinik für Innere Medizin III, Medizinische Universität Wien, Währinger Gürtel 18-20, 1090 Wien, Österreich

yvonne.winhofer-stoeckl@meduniwien.ac.at

M. Clodi

ICMR - Institute for Cardiovascular and Metabolic Research, Johannes Kepler Universität Linz, 4040 Linz, Österreich martin.clodi@jku.at
}

Abteilung für Innere Medizin, Konventhospital der Barmherzigen Brüder Linz, Seilerstätte 2, 4021 Linz, Österreich

\section{A. Kautzky-Willer}

Gender Medicine Unit, Klinische Abteilung für Endokrinologie und Stoffwechsel, Universitätsklinik für Innere Medizin III, Medizinische Universität Wien, Währinger Gürtel 18-20, 1090 Wien, Österreich alexandra.kautzky-willer@meduniwien.ac.at (z. B. Post-Transplant-Diabetes, MODY) werden angeführt. Die Auswirkungen präventiver Maßnahmen, wie die Effektivität der Lebensstilmodifikation und medikamentöse Interventionen auf die Diabetesrisikoreduktion werden in Tabellen übersichtlich zusammengefasst und können somit auch im klinischen Alltag den Patienten gut kommuniziert werden.

\section{Andere spezifische Diabetesformen und exokrine Pankreasinsuffizienz}

Im Rahmen der Zusammenlegung dieser vormals getrennten Leitlinien wurden die Themen ausführlich überarbeitet. Die Leitlinie umfasst Empfehlungen zu Diabetes bei endokrinen Erkrankungen, medikamentös-induzierte und genetische Diabetesformen sowie den neonatalen, pankreopriven und den mit Zystischer Fibrose assoziierten Diabetes. Prävalenz, Pathogenese, Klinik und Diagnostik sowie Therapie der exokrinen Pankreasinsuffizienz werden ausführlich diskutiert.

\section{Antihyperglykämische Therapie bei Diabetes mel- litus Typ 2}

In Analogie zu internationalen Empfehlungen, wurden die Ergebnisse der kardiovaskulären OutcomeStudien (CVOTs) inkludiert, die auch den neuen Algorithmus zur blutzuckersenkenden Therapie des T2DM bedingen. Demnach wird die Zweitlinientherapie nach Metformin durch die Anamnese und Klinik hinsichtlich des Vorliegens kardiovaskulärer Erkrankungen (KHK und Herzinsuffizienz) und der chronischen Niereninsuffizienz unterschieden. In den aktuellen Studien sind vor allem positive Resultate bei den GLP-1 Analoga wie auch den SGLT-2 Hemmern erhoben worden. 
Zudem wurde eine Empfehlung zur medikamentösen Therapie bei Prädiabetes und die Empfehlung zur Vitamin B12-Kontrolle unter laufender Metformintherapie ergänzt.

\section{Insulintherapie bei Diabetes mellitus Typ 2}

Die neue Leitlinie befasst sich nun ausschließlich mit der Insulintherapie bei Typ-2-Diabetes, weshalb auch die Basalinsulin-unterstützte orale Therapie (BOT) und die entsprechenden kardiovaskulären Sicherheitsdaten in dieser Leitlinie ausführlich beschrieben werden.

\section{Insulinpumpentherapie bei Kindern, Jugendli- chen und Erwachsenen}

Die Vor- und Nachteile der Insulinpumpentherapie (CSII) bei Kindern, Jugendlichen und Erwachsenen sowie die Indikationen wurden überarbeitet. Zusätzlich wird die sensorunterstützte Pumpentherapie (SuP) als wesentliche Neuheit diskutiert, der Vorteil liegt hier bei der automatischen Abschaltung der Insulinzufuhr bei Hypoglykämiegefahr.

\section{Lebensstil: Ernährung, Bewegung, Rauchent- wöhnung, Alkohol}

Die Leitlinien zur Lifestyle-Modifikation, die eine medikamentöse Therapie des Diabetes mellitus stets begleiten soll, werden in den Empfehlungen zu Bewegung, Ernährung, Rauchentwöhnung und Alkohol beschrieben.

Die Leitlinie Lebensstil: körperliche Aktivität und Training in der Prävention und Therapie des Typ 2 Diabetes mellitus wurde um das Statement zur Inaktivität und systematischen Unterbrechung von sitzender Tätigkeit nach idealerweise 30 min erweitert und standardisierte angeleitete zielgruppenspezifische Bewegungsprogramme werden empfohlen.

Die Ernährungsempfehlungen bei Diabetes mellitus wurden um ein Statement zu „low-carb high-fat (LCHF)“-Diäten und ihrer Extremform der ketogenen Diät ergänzt.

Da Rauchen und Passivrauchen sowohl die Diabetesinzidenz als auch die Wahrscheinlichkeit für Spätschäden deutlich erhöhen und auch ein moderater Alkoholkonsum bei Diabetes mellitus derzeit nicht freigesprochen werden kann, wurde die neue Leitlinie Rauchen, Alkohol und Diabetes mellitus erarbeitet.

\section{„Diabesity“ - Adipositas und Typ-2-Diabetes}

In der aktuellen Leitlinie wurden Empfehlungen überarbeitet bezüglich 1) der Effektivität der Gewichtsreduktion hinsichtlich Diabetesprävention und -therapieoptimierung, 2) des Vorteils antihyperglykämischer Substanzen mit gewichtsreduzierendem Effekt und 3) des Stellenwerts der bariatrischen Chirurgie als metabolische Therapieoption bei Diabesity aufgrund der hohen Diabetesremissionsraten von 45-95\%.

\section{Diagnostik und Therapie des Typ-1-Diabetes}

Diese neue Leitlinie beinhaltet die vormals in den Leitlinien „Autoimmunerkrankungen bei Typ 1 Diabetes“ und „Insulintherapie bei Diabetes“ beschriebenen Empfehlungen zur Diagnostik, Therapie und Begleiterkrankungen des Typ 1 Diabetes im Erwachsenenalter.

\section{Diabetes mellitus im Kindes- und Jugendalter}

Das HbAlc-Ziel bei Kindern und Jugendlichen wird nach Empfehlungen der APEDÖ (Arbeitsgruppe für pädiatrische Endokrinologie und Diabetologie Österreich) für alle Altersgruppen auf $\leq 7 \%$ (IFCC $\leq 53$ $\mathrm{mmol} / \mathrm{mol}$ ) festgelegt, vorausgesetzt dies kann ohne schwere Hypoglykämien erreicht werden. Aktuelle Daten zur Diabetes-Inzidenz wurden ergänzt. Die Indikationen für die Insulinpumpentherapie sowie die Notwendigkeit der Verwendung eines verdünnten Insulins für die Pumpe werden beschrieben.

Die regelmäßige Blutzuckermessung durch Blutzuckerbestimmung aus kapillärem Blut der Fingerbeere, mittels Flash-Glukosemessung oder kontinuierlicher subkutaner Glukosemessung (real-time CGM) sollte 6 - bis 10mal täglich stattfinden, bei Letzteren soll auch darauf geachtet werden, ob dementsprechend gescannt bzw. kalibriert wird. Auch sollen die CGM-Stellen in Analogie zu den Spritz-/ Katheterinjektionsstellen regelmäßig kontrolliert werden.

Da zahlreiche psychiatrische Komorbiditäten bei T1DM vorkommen (Essstörungen, Insulinmanipulation etc.), sollte ein Psychologe/eine Psychologin dem multidisziplinären Behandlungsteam angehören. Ein Screening auf assoziierte Autoimmunerkrankungen, vor allem Autoimmunthyreoiditis (AIT) und Zöliakie, wird bei Neumanifestation und dann alle 2 Jahre empfohlen.

Der Einsatz von ACE-Hemmern bei Kindern und Jugendlichen mit T1DM wird bei Auftreten von Mikroalbuminurie, diabetischer Nephropathie oder Hypertonie empfohlen.

\section{Gestationsdiabetes}

Die aktuelle Leitlinie enthält einen Absatz zur Prävention des Gestationsdiabetes (GDM).

Unverändert müssen alle schwangeren Frauen zwischen der 24. und 28. Schwangerschaftswoche mittels eines $75 \mathrm{~g}$ OGTT auf GDM untersucht werden; ausgenommen davon sind Frauen, bei denen bereits ein GDM oder manifester DM in der Schwangerschaft diagnostiziert wurde und Frauen nach bariatrischer Chirurgie. Letztere sollten aufgrund der Gefahr der post- 
prandialen Hypoglykämie (Dumping) mittels Erstellung eines Blutzuckerprofiles gescreent werden.

Insulin wird als Mittel der Wahl zur Therapie des GDM empfohlen und ist oralen antihyperglykämisch wirksamen Substanzen vorzuziehen.

Alle Insulinanaloga mit Ausnahme von Insulin glulisin und degludec (keine Bedenken, aber fehlende Sicherheitsdaten) sind in der Schwangerschaft zugelassen; auch das ultraschnell-wirksame Insulin aspart, das durch die rasche Resorption zur Optimierung postprandialer Hyperglykämien angewendet werden kann.

Aufgrund der nachgewiesenen positiven metabolischen Auswirkungen auf die Mutter und das Kind wird Stillen sehr empfohlen.

\section{Gravidität bei vorbestehendem Diabetes}

Aufgrund der zunehmenden Adipositasprävalenz ist die Anzahl an Frauen mit manifestem Typ-2-Diabetes in den letzten Jahren deutlich angestiegen, sodass nun in dieser Leitlinie sowohl auf Frauen mit T1DM als auch T2DM in der Schwangerschaft eingegangen wird. Der Schwangerschaftsplanung kommt hierbei eine entscheidende Rolle $\mathrm{zu}$, um die diabetische Embryopathie als Folge der Hyperglykämie im ersten Trimester zu verhindern. Ein präkonzeptionelles HbAlc $<6,5 \%$ (zumindest $<7 \%$ ) ist anzustreben.

Sowohl die Basis-Bolus-Therapie als auch die Insulinpumpentherapie werden als weitgehend gleichwertig angesehen, wobei auf komplexere Therapiekonzepte bereits bei Kinderwunsch und nicht erst in der Schwangerschaft umgestellt werden soll. Seit der CONCEPTT-Studie ist nachgewiesen, dass der Einsatz der kontinuierlichen Glukosemessung mittels CGMS im Vergleich zur kapillären Messungen mit einem besseren neonatalen Outcome assoziiert ist und somit absolut zu empfehlen ist.

Die Empfehlungen zu den verschiedenen Insulinen sowie einer Metformintherapie bei übergewichtigen Frauen entsprechen jenen in der Leitlinie Gestationsdiabetes, siehe oben.

\section{Diabetesschulung und -beratung bei Erwachse- nen mit Diabetes}

Aufgrund der technischen Innovationen auf dem Gebiet der Diabetestherapie sollen Programme zum Umgang mit technischen Neuerungen wie Glukosesensor-Systemen und Insulinpumpen zunehmend Einsatz finden.

Auch Diabetes-Apps (z.B. MySugr) und DiabetesPortale (z. B. Diabetes-Patientenfuchs) können Unterstützung bieten.

Spezifisch ethnisch-kulturelle Aspekte sollten in der Schulung berücksichtigt werden, um eine nachhaltige Verbesserung der Stoffwechsellage zu erreichen. Disease Management Programme (DMP, „Therapie aktiv“) sind in der Diabetesbetreuung effektiv.

\section{Blutzuckerselbstkontrolle}

Diese Leitlinie wurde um Empfehlungen zum Einsatz von CGMS und Flash-Glukosemessung erweitert.

\section{Kontinuierliche Glukosemessung (CGM - Contin- uous Glucose Monitoring) bei Diabetes mellitus}

Aufgrund rezenter technischer Innovationen und der Verfügbarkeit zahlreicher CGM-Systeme wurde diese Leitlinie entsprechend aktualisiert. Vor- und Nachteile der intermittently scanned CGM (isCGM oder Flash Glukosemessung FGM) und der real-time CGM ( $r$ CGM) werden diskutiert und alle derzeit verfügbaren Systeme aufgelistet. In vielen Studien konnte mittlerweile der positive Effekt der CGM auf die Stoffwechseleinstellung (HbAlc-Senkung) nachgewiesen werden. Indikationen werden aufgelistet, die beim Ansuchen auf Erstattung helfen sollen.

\section{Individualisierung der antihypertensiven Therapie bei Patienten mit Diabetes mellitus}

Der Ziel-Blutdruck bei Patienten mit Diabetes mellitus soll aufgrund der aktuellen Datenlage (Ergebnisse der großen prospektiven, randomisierten Studien) individuell aufgrund des Patientenalters und der Komorbiditäten (vor allem kardiovaskuläre Ereignisse) festgelegt werden und entsprechende Richtwerte werden tabellarisch genannt. Ein Zielblutdruck um 130/80 mm Hg dürfte nach derzeitiger Evidenz jener sein, der mit der relativ besten Prävention von vaskulären Komplikationen und mit der größten Sicherheit einhergeht. Hinsichtlich der medikamentösen Therapie sollen neben Antihypertensiva auch antihyperglykämische Substanzen mit nachgewiesener blutdrucksenkender Wirkung zum Einsatz kommen. Dazu zählen SGLT-2-Hemmer, GLP-1-Rezeptoragonisten und Pioglitazon.

\section{Lipide: Diagnostik und Therapie bei Diabetes mel- litus}

In Anlehnung an die ESC- und AACE/ACE-Leitlinien werden die LDL- bzw. Non-HDL-Cholesterin-Zielwerte je nach kardiovaskulärem Risiko (lt. ESC-Definition) empfohlen. Die medikamentöse Therapie folgt einem sequentiellen Schema, beginnend mit einer Statin-Therapie, bei Nicht-Erreichen sollen Ezetimib und im Weiteren PCSK9-Hemmer zum Einsatz kommen. Dass Letztere auch bei Patienten mit Diabetes mellitus einen kardiovaskulären Benefit aufweisen, konnten die kardiovaskulären Outcome-Studien, FOURIER und ODYSSEY OUTCOMES, nachweisen.

\section{Thrombozytenaggregationshemmer}

Mit Bezug auf die 2018 publizierte Studie mit über 15.000 Teilnehmern zum Einsatz der Acetylsalicylsäu- 
re (ASS) sollte diese in der Primärprävention nur nach entsprechender Nutzen-Risikoabwägung eingesetzt werden (die erreichte Senkung der kardiovaskulären Ereignisse entspricht etwa dem Anstieg der gastrointestinalen Blutungen). Eine klare Indikation zur Thrombozyten-Aggregationshemmung besteht bei Patienten mit Diabetes in der Sekundärprävention (62-162 mg/Tag).

\section{Diabetische Neuropathie und diabetischer Fuß}

Die LL „Diabetische Neuropathie“ und „Diabetischer Fuß“ wurden zu einer gemeinsamen Leitlinie zusammengefasst.

\section{Diabetische Nierenerkrankung}

In dieser Leitlinie wurden aktuelle epidemiologische Daten ergänzt. Zur Bestimmung der Nierenfunktion wird empfohlen, eine Schätzformel (MDRD, CKD-EPI) zur Berechnung der eGFR heranzuziehen. Renale Effekte der blutzuckersenkenden Medikamente werden beschrieben sowie die sichere Anwendung und Dosierung blutzuckersenkender Medikamente bei eingeschränkter Nierenfunktion.

Hinsichtlich der Therapie-Zielwerte bleiben Hbalcund Blutdruckziel gleich; LDL-Cholesterin-Zielwerte werden nun angeführt. Die Reduktion der Salzzufuhr unter 5-6g/Tag kann nicht mehr generell empfohlen werden.

\section{Diagnose, Therapie und Verlaufskontrolle der dia- betischen Augenerkrankung}

Die OCT-Angiographie wurde als diagnostische Methode zur Lokalisierung von avaskulären Arealen sowie als Nachweis von vaskulären Alterationen inkl. Neovaskularisationen ergänzt. Zur Behandlung der proliferativen diabetischen Retinopathie sind neben der panretinalen Laserkoagulation und der Glaskörper-/Netzhautchirurgie nun auch antiVEGFIVOM (in Kombination) zugelassen.

\section{Koronare Herzkrankheit und Herzinsuffizienz}

In der Diagnostik der Herzinsuffizienz kommt dem NT-proBNP eine immer größere Rolle zu (Rule-Out bei proBNP $<125 \mathrm{pg} / \mathrm{ml}$ ) und kann in Verbindung mit der Klinik und Echokardiographie auch der Diagnose der HFpEF dienen.

Die CT-Angiographie zum Nachweis bzw. Ausschluss einer KHK kann auch bei asymptomatischen Patienten in Erwägung gezogen werden, da sie bei Patienten mit Diabetes mellitus derzeit die beste Sensitivität unter den nicht invasiven Untersuchungen hat.

In Analogie zur Leitlinie „Antihyperglykämische Therapie bei Diabetes mellitus Typ 2" werden die Empfehlungen zur glukosesenkenden Therapie bei
KHK und/oder Herzinsuffizienz durch die kardiovaskulären Endpunktstudien geprägt, wobei positive Effekte derzeit für Empagliflozin, Dapagliflozin, Canagliflozin und Liraglutid nachgewiesen sind.

\section{Diagnose und Management der Osteoporose bei Diabetes mellitus}

Diese neue Leitlinie wurde gemeinsam mit der Österreichischen Gesellschaft für Knochen- und Mineralstoffwechsel erarbeitet und bietet Empfehlungen zur Diagnostik und Therapie der Osteoporose bei Patienten mit Diabetes. Des Weiteren werden die Effekte der antihyperglykämischen Medikamente auf den Knochen zusammengefasst.

\section{Psychische Erkrankungen und Diabetes mellitus}

Der Zusammenhang zwischen Diabetes und psychischen Erkrankungen sowie die Stoffwechseleffekte psychopharmakologischer Therapien werden ausführlich dargestellt und durch aktuelle Literatur belegt. Die Ursachen und Auswirkungen von „diabetes distress“ werden beschrieben. In Analogie zur Forderung der Amerikanischen Diabetes Gesellschaft sollte auch in Österreich eine umfassende psychosoziale Betreuung in die Diabetesbehandlung integriert und für alle Diabetiker zugänglich gemacht werden.

\section{Therapie der akuten diabetischen Stoffwechsel- entgleisungen bei Erwachsenen}

Diese neue Leitlinie gibt Empfehlungen zur Diagnose und Therapie der ketoazidotischen (DKA) und hyperglykämisch-hyperosmolaren (HHS) Stoffwechselentgleisung, beides lebensbedrohliche Komplikationen bei Patienten mit Diabetes.

\section{Diabetesmanagement im Krankenhaus}

Diese neue Leitlinie enthält Empfehlung zum Management stationärer Patienten mit Diabetes mellitus hinsichtlich Blutzuckerzielbereiche, orale antihyperglykämische Therapie und Insulintherapie sowie Spezialsituationen wie Glukokortikoidtherapie und der Anwendung der Diabetestechnologie im stationären Setting.

\section{Operation und Diabetes mellitus}

Dieses Positionspapier wurde speziell für die perioperative Betreuung von Patienten mit Diabetes mellitus erarbeitet. Die präoperative Evaluierung und Notwendigkeit der optimalen Stoffwechselkontrolle werden ausführlich beschrieben. Hinsichtlich der medikamentösen Therapie ist hervorzuheben, dass Metformin und SGLT-2-Hemmer präoperativ pausiert $(24 \mathrm{~h}$ bei elektivem Standardeingriff und $48 \mathrm{~h}$ bei großem Eingriff) und postoperativ erst nach Stabilisierung der 
klinischen Situation wiederbegonnen werden sollen. Bei komplexen Therapieregimen und/oder diabetischen Spätkomplikationen soll das Hinzuziehen eines Diabetologen angedacht werden.

\section{Therapie bei kritisch kranken Patienten}

Diese Leitlinie gibt Empfehlungen zur Therapie bei kritisch kranken Patienten. Hier ist die Datenlage stabil ohne wesentliche neue Erkenntnisse in den letzten Jahren.

\section{Migration und Diabetes}

Diese Leitlinie wurde um aktuelle epidemiologische Daten und Empfehlungen zur Ernährung allgemein sowie zum Fasten (Ramadan) erweitert. Für letzteres gibt es auch Empfehlungen zur Anpassung der antihyperglykämischen Therapie (orale Antidiabetika und Insulin).

\section{Geschlechtsspezifische Aspekte bei Prädiabetes und Diabetes mellitus - klinische Empfehlungen}

Die aktuelle Leitlinie enthält neue epidemiologische Daten zu den verschiedenen Diabetesformen inkl. Prädiabetes. Problematik und mögliche Therapieansätze der sexuellen Dysfunktion bei Frauen werden diskutiert. Obwohl sich die blutzuckersenkenden Effekte der antihyperglykämischen Substanzen nicht wesentlich unterscheiden, werden unterschiedliche
Nebenwirkungen bei Männern und Frauen beobachtet und diese tabellarisch zusammengefasst.

\section{Geriatrische Aspekte bei Diabetes mellitus}

Da Begleiterscheinungen wie Frailty („Gebrechlichkeit“), Sarkopenie, kognitive Beeinträchtigung und Demenz den Therapieverlauf bei geriatrischen Patienten mit Diabetes mellitus wesentlich beeinflussen, wird ein Screening mittels Fragebögen empfohlen.

Tab. 3 liefert Ziel-Korridore (HbAlc, RR, Lipide) für ältere Patienten in Abhängigkeit ihrer funktionellen Beeinträchtigung. Therapieziele (Blutdruck) wurden anhand neuer Evidenz aktualisiert. Die Insulintherapie sowohl bei Typ-2- als auch Typ-1-Diabetes im höheren Alter wird nun ausführlicher diskutiert.

Interessenkonflikt Y. Winhofer-Stöckl hat von folgenden Firmen, die teils auch fördernde Mitglieder der ÖDG sind, Honorare für Vorträge und Beratung erhalten: Amgen, AstraZeneca, Boehringer Ingelheim, Eli Lilly, Merck Sharp \& Dohme, Novartis, NovoNordisk, Sanofi. M. Clodi hat Vortragshonorare und Honorare für Beratungstätigkeit von folgenden Firmen erhalten: Eli Lilly, AstraZeneca, Boehringer Ingelheim, Amgen, Sanofi, Novo Nordisk, Servier. A. KautzkyWiller hat von folgenden Unternehmen, die auch fördernde Mitglieder der ÖDG sind, Forschungsunterstützungen und/ oder Honorare erhalten: AstraZeneca, Amgen, Boehringer Ingelheim, Eli Lilly, Merck Sharp \& Dohme, Novartis, Novo Nordisk, Roche, sanofi-aventis, Servier, Takeda.

Hinweis des Verlags Der Verlag bleibt in Hinblick auf geografische Zuordnungen und Gebietsbezeichnungen in veröffentlichten Karten und Institutsadressen neutral. 